\title{
Power series inequalities via Young's inequality with applications
}

Alawiah Ibrahim ${ }^{1,3^{*}}$, Sever S Dragomir ${ }^{1,2}$ and Maslina Darus ${ }^{3}$

\section{"Correspondence:}

alawiah.ibrahim@live.vu.edu.au

${ }^{1}$ School of Engineering and Science, Victoria University, P.O. Box 14428,

Melbourne, 8001, Australia

${ }^{3}$ School of Mathematical Sciences,

Faculty of Science and Technology, Universiti Kebangsaan Malaysia,

Bangi, Selangor 43600, Malaysia Full list of author information is available at the end of the article

\begin{abstract}
In this paper, we establish some inequalities for power series with real coefficients by utilizing Young's inequality for sequences of complex numbers. Some applications for special functions such as polylogarithm, hypergeometric and Bessel functions are also presented.
\end{abstract}

MSC: 26D15

Keywords: Young's inequality; Hölder's inequality; power series

\section{Introduction}

Let $a_{k}, b_{k} \in \mathbb{C}, k \in\{1,2, \ldots, n\}, p>1$ and let $q$ satisfy $\frac{1}{p}+\frac{1}{q}=1$. Then the classical Hölder's inequality $[1, \mathrm{pp} .19-21]$ states that

$$
\left|\sum_{k=1}^{n} a_{k} b_{k}\right| \leq\left(\sum_{k=1}^{n}\left|a_{k}\right|^{p}\right)^{\frac{1}{p}}\left(\sum_{k=1}^{n}\left|b_{k}\right|^{q}\right)^{\frac{1}{q}}
$$

with equality holds if and only if the sequences $\left\{\left|a_{k}\right|^{p}\right\}$ and $\left\{\left|b_{k}\right|^{q}\right\}$ for $k \in\{1,2, \ldots, n\}$ are proportional and the $\arg \left(a_{k} b_{k}\right)$ is independent of $k$. The inequality (1.1) is reversed if $p<1$.

The weighted version of Hölder's inequality also holds, namely

$$
\left|\sum_{k=1}^{n} p_{k} a_{k} b_{k}\right| \leq\left(\sum_{k=1}^{n} p_{k}\left|a_{k}\right|^{p}\right)^{\frac{1}{p}}\left(\sum_{k=1}^{n} p_{k}\left|b_{k}\right|^{q}\right)^{\frac{1}{q}},
$$

where $p_{k} \geq 0, a_{k}, b_{k} \in \mathbb{C}, k \in\{1,2, \ldots, n\}, p>1, \frac{1}{p}+\frac{1}{q}=1$.

Tolsted in [2] (see also [3, p.457], [4, pp.63-64]) showed that Hölder's inequality (also known in the literature as the Rogers inequality) can be easily proved by using Young's inequality [5], namely

$$
\frac{1}{q} x^{q}+\frac{1}{p} y^{p} \geq x y
$$

for any positive numbers $x, y$ and $p>1$ with $\frac{1}{p}+\frac{1}{q}=1$. Equality holds in (1.3) if and only if $x^{q}=y^{p}$. For other applications and extensions of Young's inequality, see [6, 7], [8, pp.379389] and references therein.

It is well known that Hölder's inequality is one of the most important inequalities in real and complex analysis. For example, the celebrated Cauchy-Bunyakovsky-Schwarz (CBS)

\section{Springer}

(c) 2013 Ibrahim et al.; licensee Springer. This is an Open Access article distributed under the terms of the Creative Commons Attribution License (http://creativecommons.org/licenses/by/2.0), which permits unrestricted use, distribution, and reproduction in any medium, provided the original work is properly cited. 
inequality (see [9, p.16], [8, p.83]) is a special case of Hölder's inequality (1.1) for $p=q=2$. Some other inequalities such as Minkowski's inequality can be proved by using Hölder's inequality.

Various extensions, generalizations, refinements, etc. of Hölder's inequality have been obtained by several authors (see [10-20] and references therein). For instance, it comes to our attention that an interesting generalizations of Hölder's inequality (1.1) by utilizing Young's inequality (1.3), which was established by Dragomir and Sándor in [21] (see also [22, pp.10-16]), is as follows:

$$
\begin{aligned}
& \sum_{k=1}^{n} p_{k}\left|x_{k} y_{k}\right| \sum_{k=1}^{n} q_{k}\left|x_{k} y_{k}\right| \\
& \quad \leq \frac{1}{p} \sum_{k=1}^{n} p_{k}\left|x_{k}\right|^{p} \sum_{k=1}^{n} q_{k}\left|y_{k}\right|^{p}+\frac{1}{q} \sum_{k=1}^{n} q_{k}\left|x_{k}\right|^{q} \sum_{k=1}^{n} p_{k}\left|y_{k}\right|^{q}
\end{aligned}
$$

for $x_{k}, y_{k} \in \mathbb{C}, p_{k}, q_{k} \geq 0, k \in\{1,2, \ldots, n\}$ and $p, q>1$ with $\frac{1}{p}+\frac{1}{q}=1$.

If now we consider an analytic function defined by the power series

$$
f(z)=\sum_{n=0}^{\infty} a_{n} z^{n}
$$

with real coefficients and convergent on the disk $D(0, R), R>0$ and apply the weighted version of Hölder's inequality (1.2), then we can state that

$$
\begin{aligned}
|f(x y)| & =\left|\sum_{n=0}^{\infty} a_{n} x^{n} y^{n}\right| \\
& \leq\left(\sum_{n=0}^{\infty}\left|a_{n}\right||x|^{p n}\right)^{\frac{1}{p}}\left(\sum_{n=0}^{\infty}\left|a_{n}\right||y|^{q n}\right)^{\frac{1}{q}}=f_{A}^{\frac{1}{p}}\left(|x|^{p}\right) f_{A}^{\frac{1}{q}}\left(|y|^{q}\right)
\end{aligned}
$$

for any $x, y \in \mathbb{C}$ with $x y,|x|^{p},|y|^{q} \in D(0, R)$ and $f_{A}(z)$ is a new power series defined by $\sum_{n=0}^{\infty}\left|a_{n}\right| z^{n}$, where $a_{n}=\left|a_{n}\right| \operatorname{sgn}\left(a_{n}\right)$ with $\operatorname{sgn}(x)$ is the real signum function defined to be 1 if $x>0,-1$ if $x<0$ and 0 if $x=0$. The power series $f_{A}(z)$ have the same radius of convergence as the original power series $f(z)$.

Motivated by the above results (1.6), (1.4) and the results from [21], and utilizing Young's inequality, we established in this paper some inequalities for functions defined by power series with real coefficients. Particular examples that are related to some fundamental complex functions such as the exponential, logarithm, trigonometric and hyperbolic functions are presented. Applications for some special functions such as polylogarithm, hypergeometric and Bessel functions for the first kind are presented as well.

\section{Some inequalities via Young's inequality}

On utilizing Young's inequality (1.3) for power series with real coefficients, we establish the following result.

Theorem 1 Let $f(z)=\sum_{n=0}^{\infty} p_{n} z^{n}$ and $g(z)=\sum_{n=0}^{\infty} q_{n} z^{n}$ be two power series with real coeffcients and convergent on the open disk $D(0, R), R>0$. If $p>1, \frac{1}{p}+\frac{1}{q}=1$ and $x, y \in \mathbb{C}, x, y \neq 0$ 
so that $x y,|x|^{p},|x|^{q},|y|^{p},|y|^{q} \in D(0, R)$, then

$$
\frac{1}{p} g_{A}\left(|x|^{p}\right) f_{A}\left(|y|^{p}\right)+\frac{1}{q} f_{A}\left(|x|^{q}\right) g_{A}\left(|y|^{q}\right) \geq|f(x y) g(x y)|
$$

and

$$
\frac{1}{p} g_{A}\left(|x|^{p}\right) f_{A}\left(|y|^{q}\right)+\frac{1}{q} f_{A}\left(|x|^{q}\right) g_{A}\left(|y|^{p}\right) \geq\left|f\left(x|y|^{q-1}\right) g\left(x|y|^{p-1}\right)\right| .
$$

Proof If we choose $x=|x|^{j}|y|^{k}, y=|x|^{k}|y|^{j}, j, k \in\{0,1,2, \ldots, n\}$ in (1.3), then we have

$$
p|x|^{q j}|y|^{q k}+q|x|^{p k}|y|^{p j} \geq p q|x y|^{j}|x y|^{k}
$$

for any $j, k \in\{0,1,2, \ldots, n\}$.

Now, if we multiply this inequality (2.3) with positive quantities $\left|p_{j}\right|\left|q_{k}\right|$ and summing over $j$ and $k$ from 0 to $n$, then we derive

$$
\begin{aligned}
& p \sum_{j=0}^{n}\left|p_{j}\right||x|^{q j} \sum_{k=0}^{n}\left|q_{k}\right||y|^{q k}+q \sum_{k=0}^{n}\left|q_{k}\right||x|^{p k} \sum_{j=0}^{n}\left|p_{j}\right||y|^{p j} \\
& \geq p q\left|\sum_{j=0}^{n} p_{j}(x y)^{j} \sum_{k=0}^{n} q_{k}(x y)^{k}\right| .
\end{aligned}
$$

Since all the series whose partial sums are involved in inequality (2.4) are convergent on the disk $D(0, R)$, taking the limit as $n \rightarrow \infty$ in (2.4), we deduce the desired result (2.1).

Further, if we choose in (1.3), $x=\frac{\mid x j^{j}}{|y|^{j}}, y=\frac{|x|^{k}}{|y|^{k}}$, then we get

$$
p\left(\frac{|x|^{j}}{|y|^{j}}\right)^{q}+q\left(\frac{|x|^{k}}{|y|^{k}}\right)^{p} \geq p q \frac{|x|^{j}}{|y|^{j}} \frac{|x|^{k}}{|y|^{k}}
$$

for any $|y|^{j},|y|^{k} \neq 0, j, k \in\{0,1,2, \ldots, n\}$.

Simplifying (2.5), we obtain that

$$
\begin{aligned}
p|x|^{q j}|y|^{p k}+q|x|^{p k}|y|^{q j} & \geq p q|x|^{j}|y|^{(q-1) j}|x|^{k}|y|^{(p-1) k} \\
& =p q\left|\left(x|y|^{q-1}\right)^{j}\left(x|y|^{p-1}\right)^{k}\right|
\end{aligned}
$$

for any $j, k \in\{0,1,2, \ldots, n\}$.

Multiplying (2.6) by $\left|p_{j}\right|\left|q_{k}\right| \geq 0, j, k \in\{0,1,2, \ldots, n\}$ and summing over $j$ and $k$ from 0 to $n$, we get

$$
\begin{gathered}
p \sum_{j=0}^{n}\left|p_{j}\right||x|^{q j} \sum_{k=0}^{n}\left|q_{k}\right||y|^{p k}+q \sum_{k=0}^{n}\left|q_{k}\right||x|^{p k} \sum_{j=0}^{n}\left|p_{j}\right||y|^{q j} \\
\geq p q\left|\sum_{j=0}^{n} p_{j}\left(x|y|^{q-1}\right)^{j} \sum_{k=0}^{n} q_{k}\left(x|y|^{p-1}\right)^{k}\right| .
\end{gathered}
$$

Since all the series whose partial sums are involved in inequality (2.7) are convergent on the disk $D(0, R)$, letting $n \rightarrow \infty$ in (2.7), we deduce the desired inequality (2.2). 
The following particular case is of interest.

Corollary 1 If $g(z)=f(z)$ in (2.1) and (2.2), then

$$
\frac{1}{p} f_{A}\left(|x|^{p}\right) f_{A}\left(|y|^{p}\right)+\frac{1}{q} f_{A}\left(|x|^{q}\right) f_{A}\left(|y|^{q}\right) \geq|f(x y)|^{2}
$$

and

$$
\frac{1}{p} f_{A}\left(|x|^{p}\right) f_{A}\left(|y|^{q}\right)+\frac{1}{q} f_{A}\left(|x|^{q}\right) f_{A}\left(|y|^{p}\right) \geq\left|f\left(x|y|^{q-1}\right) f\left(x|y|^{p-1}\right)\right|,
$$

respectively, where $p>1, \frac{1}{p}+\frac{1}{q}=1$ and $x, y \neq 0$ with $x y,|x|^{p},|x|^{q},|y|^{p},|y|^{q} \in D(0, R)$. In particular, if $y=x$ in (2.8) and (2.9), then we have

$$
\frac{1}{p} f_{A}^{2}\left(|x|^{p}\right)+\frac{1}{q} f_{A}^{2}\left(|x|^{q}\right) \geq\left|f\left(x^{2}\right)\right|^{2}
$$

and

$$
f_{A}\left(|x|^{p}\right) f_{A}\left(|x|^{q}\right) \geq\left|f\left(\operatorname{sgn}(x)|x|^{q}\right) f\left(\operatorname{sgn}(x)|x|^{p}\right)\right|
$$

for any $x \in \mathbb{C}, x \neq 0$ with $x^{2},|x|^{p},|x|^{q} \in D(0, R)$ and $\operatorname{sgn}(x)$ is the complex signum function defined to be $\frac{x}{|x|}$ if $x \neq 0$ and 0 if $x=0$.

Remark 1 In the particular case $p=q=2$ in (2.8) and (2.9), we get the inequalities

$$
f_{A}\left(|x|^{2}\right) f_{A}\left(|y|^{2}\right) \geq|f(x y)|^{2}
$$

and

$$
f_{A}\left(|x|^{2}\right) f_{A}\left(|y|^{2}\right) \geq|f(x|y|)|^{2},
$$

respectively, for any $x, y \in \mathbb{C}$ with $x y,|x|^{2},|y|^{2} \in D(0, R)$.

Some applications to particular functions of interest are as follows.

(1) If we apply inequalities (2.8) and (2.9) to the function $f(z)=\frac{1}{1-z}=\sum_{n=0}^{\infty} z^{n}, z \in D(0,1)$, then we get

$$
\frac{1}{p\left(1-|x|^{p}\right)\left(1-|y|^{p}\right)}+\frac{1}{q\left(1-|x|^{q}\right)\left(1-|y|^{q}\right)} \geq \frac{1}{|1-x y|^{2}}
$$

and

$$
\begin{aligned}
& \frac{1}{p\left(1-|x|^{p}\right)\left(1-|y|^{q}\right)}+\frac{1}{q\left(1-|x|^{q}\right)\left(1-|y|^{p}\right)} \\
& \geq \frac{1}{\left.|1-x| y\right|^{q-1}|| 1-x|y|^{p-1} \mid},
\end{aligned}
$$

respectively, for any $x, y \in \mathbb{C}$ with $x, y \neq 0, x y,|x|^{p},|x|^{q},|y|^{p},|y|^{q} \in D(0,1)$ and $p>1, \frac{1}{p}+\frac{1}{q}=1$. 
(2) If we apply inequalities (2.8) and (2.9) to the function $f(z)=\exp (z)=\sum_{n=0}^{\infty} \frac{1}{n !} z^{n}, z \in \mathbb{C}$, then we can state that

$$
\frac{1}{p} \exp \left(|x|^{p}+|y|^{p}\right)+\frac{1}{q} \exp \left(|x|^{q}+|y|^{q}\right) \geq|\exp (x y)|^{2}
$$

and

$$
\begin{aligned}
& \frac{1}{p} \exp \left(|x|^{p}+|y|^{q}\right)+\frac{1}{q} \exp \left(|x|^{q}+|y|^{p}\right) \\
& \geq\left|\exp \left(x|y|^{q-1}+x|y|^{p-1}\right)\right|
\end{aligned}
$$

respectively, for any $x, y \in \mathbb{C}$ and $p>1$ with $\frac{1}{p}+\frac{1}{q}=1$.

(3) If we apply the function $f(z)=\ln \left(\frac{1}{1-z}\right)=\sum_{n=0}^{\infty} \frac{1}{n} z^{n}, z \in D(0,1)$, then from (2.8) and (2.9) we have

$$
\begin{aligned}
& \frac{1}{p} \ln \left(1-|x|^{p}\right) \ln \left(1-|y|^{p}\right)+\frac{1}{q} \ln \left(1-|x|^{q}\right) \ln \left(1-|y|^{q}\right) \\
& \quad \geq|\ln (1-x y)|^{2}
\end{aligned}
$$

and

$$
\begin{aligned}
& \frac{1}{p} \ln \left(1-|x|^{p}\right) \ln \left(1-|y|^{q}\right)+\frac{1}{q} \ln \left(1-|x|^{q}\right) \ln \left(1-|y|^{p}\right) \\
& \quad \geq\left|\ln \left(1-x|y|^{q-1}\right) \ln \left(1-x|y|^{p-1}\right)\right|,
\end{aligned}
$$

respectively, for any $x, y \in \mathbb{C}$ with $x, y \neq 0,|x|^{p},|x|^{q},|y|^{p},|y|^{q} \in D(0,1)$ and $p>1, \frac{1}{p}+\frac{1}{q}=1$.

(IV) Also, if we consider the function $f(z)=\sin (z)=\sum_{n=0}^{\infty} \frac{(-1)^{n}}{(2 n+1) !} z^{2 n+1}, z \in \mathbb{C}$, then, obviously, we have $f_{A}(z)=\sinh (z), z \in \mathbb{C}$. Applying inequalities (2.8) and (2.9) to this function, we get

$$
\frac{1}{p} \sinh \left(|x|^{p}\right) \sinh \left(|y|^{p}\right)+\frac{1}{q} \sinh \left(|x|^{q}\right) \sinh \left(|y|^{q}\right) \geq|\sin (x y)|^{2}
$$

and

$$
\begin{aligned}
& \frac{1}{p} \sinh \left(|x|^{p}\right) \sinh \left(|y|^{q}\right)+\frac{1}{q} \sinh \left(|x|^{q}\right) \sinh \left(|y|^{p}\right) \\
& \quad \geq\left|\sin \left(x|y|^{q-1}\right) \sin \left(x|y|^{p-1}\right)\right|
\end{aligned}
$$

respectively, for $x, y \in \mathbb{C}$ and $p>1, \frac{1}{p}+\frac{1}{q}=1$.

Similar results can be obtained for $\cosh (x)$ as well.

The following result also holds.

Theorem 2 Let $f(z)$ and $g(z)$ be as in Theorem 1 . Then one has the inequalities

$$
\frac{1}{p} g_{A}\left(|x|^{p}\right) f_{A}\left(|y|^{q}\right)+\frac{1}{q} f_{A}\left(|x|^{p}\right) g_{A}\left(|y|^{q}\right) \geq\left|f\left(|x|^{p-1}|y|^{q-1}\right) g(x y)\right|
$$


and

$$
\frac{1}{p} f_{A}\left(|x|^{p}\right) g_{A}\left(|y|^{2}\right)+\frac{1}{q} g_{A}\left(|x|^{2}\right) f_{A}\left(|y|^{q}\right) \geq\left|f(x y) g\left(|x|^{\frac{2}{q}}|y|^{\frac{2}{p}}\right)\right| .
$$

Proof If we choose in (1.3), $x=\frac{|y|^{k}}{|y|^{j}}, y=\frac{|x|^{k}}{|x|^{j}},|x|^{j},|y|^{j} \neq 0, j, k \in\{0,1,2, \ldots, n\}$, we have

$$
\begin{aligned}
p|y|^{q k}|x|^{p j}+q|x|^{p k}|y|^{q j} & \geq p q|x|^{(p-1) j}|y|^{(q-1) j}|x y|^{k} \\
& =p q\left|\left(|x|^{p-1}|y|^{q-1}\right)^{j}(x y)^{k}\right|
\end{aligned}
$$

for any $j, k \in\{0,1,2, \ldots, n\}$.

Multiplying (2.12) with $\left|p_{j}\right|\left|q_{k}\right| \geq 0$ and summing over $j$ and $k$ from 0 to $n$, we obtain that

$$
\begin{gathered}
p \sum_{k=0}^{n}\left|q_{k}\right||y|^{q k} \sum_{j=0}^{n}\left|p_{j}\right||x|^{p j}+q \sum_{k=0}^{n}\left|q_{k}\right||x|^{p k} \sum_{j=0}^{n}\left|p_{j}\right||y|^{q j} \\
\geq p q\left|\sum_{j=0}^{n} p_{j}\left(|x|^{p-1}|y|^{q-1}\right)^{j} \sum_{k=0}^{n} q_{k}(x y)^{k}\right| .
\end{gathered}
$$

From (1.3), we also have the inequality

$$
\begin{aligned}
& p \sum_{k=1}^{n}\left|q_{k}\right||x|^{2 k} \sum_{j=1}^{n}\left|p_{j}\right||y|^{q j}+q \sum_{j=1}^{n}\left|p_{j}\right||x|^{p j} \sum_{k=1}^{n}\left|q_{k}\right||y|^{2 k} \\
& \geq p q\left|\sum_{j=1}^{n} p_{j}(x y)^{j} \sum_{k=1}^{n} q_{k}\left(|x|^{\frac{2}{q}}|y|^{\frac{2}{p}}\right)^{k}\right|
\end{aligned}
$$

for any $x, y \in C, p>1, \frac{1}{p}+\frac{1}{q}=1$, which was obtained by choosing $x=|x|^{\frac{2}{q} k}|y|^{j}, y=|x|^{j}|y|^{\frac{2}{p} k}$ and repeating the same method as above.

Now, since all the series whose partial sums are involved in inequalities (2.13) and (2.14) are convergent on the disk $D(0, R)$, by letting $n \rightarrow \infty$ in (2.13) and (2.14), respectively, we deduce the desired inequalities, i.e., (2.10) and (2.11).

Corollary 2 If $g(z)=f(z)$ in (2.10) and (2.11), then

$$
f_{A}\left(|x|^{p}\right) f_{A}\left(|y|^{q}\right) \geq\left|f(x y) f\left(|x|^{p-1}|y|^{q-1}\right)\right|
$$

and

$$
\frac{1}{p} f_{A}\left(|x|^{p}\right) f_{A}\left(|y|^{2}\right)+\frac{1}{q} f_{A}\left(|x|^{2}\right) f_{A}\left(|y|^{q}\right) \geq\left|f(x y) f\left(|x|^{\frac{2}{q}}|y|^{\frac{2}{p}}\right)\right|,
$$

respectively, where $p>1, \frac{1}{p}+\frac{1}{q}=1$ and $x, y \neq 0$ with $x y,|x|^{2},|x|^{p},|x|^{\frac{2}{q}},|y|^{2},|y|^{q},|y|^{\frac{2}{p}} \in D(0, R)$. In particular, if $y=x$ in (2.15) and (2.16), then we have

$$
f_{A}\left(|x|^{p}\right) f_{A}\left(|x|^{q}\right) \geq\left|f\left(x^{2}\right) f\left(|x|^{p q-2}\right)\right|
$$


and

$$
f_{A}\left(|x|^{2}\right)\left[\frac{1}{p} f_{A}\left(|x|^{p}\right)+\frac{1}{q} f_{A}\left(|x|^{q}\right)\right] \geq\left|f\left(x^{2}\right) f\left(|x|^{2}\right)\right|
$$

for $x \neq 0$ with $x^{2},|x|^{2},|x|^{p},|x|^{q} \in D(0, R)$.

Remark 2 In the particular case $p=q=2$ in (2.15) or (2.16), we get the inequality

$$
f_{A}\left(|x|^{2}\right) f_{A}\left(|y|^{2}\right) \geq|f(x y) f(|x y|)|
$$

for any $x, y \in \mathbb{C}$ with $x y,|x y|,|x|^{2},|y|^{2} \in D(0, R)$.

In what follows, we provide some applications of inequalities (2.15) and (2.16) to particular functions of interest.

(1) If we apply inequalities (2.15) and (2.16) to the function $f(z)=\frac{1}{1-z}, z \in D(0,1)$, then we get

$$
\left.|1-x y||1-| x\right|^{p-1}|y|^{q-1} \mid \geq\left(1-|x|^{p}\right)\left(1-|y|^{q}\right)
$$

and

$$
\begin{aligned}
& \frac{1}{p\left(1-|x|^{p}\right)\left(1-|y|^{2}\right)}+\frac{1}{q\left(1-|x|^{2}\right)\left(1-|y|^{q}\right)} \\
& \quad \geq \frac{1}{\left.|1-x y||1-| x\right|^{\frac{2}{q}}|y|^{\frac{2}{p}} \mid},
\end{aligned}
$$

respectively, where $p>1, \frac{1}{p}+\frac{1}{q}=1$ and $x, y \neq 0$ with $x y,|x|^{2},|x|^{p},|x|^{\frac{1}{q}},|y|^{2},|y|^{q},|y|^{\frac{1}{p}} \in$ $D(0,1)$.

(2) If we apply inequalities (2.15) and (2.16) to the function $f(z)=\exp (z), z \in \mathbb{C}$, then we can state that

$$
\exp \left(|x|^{p}+|y|^{q}\right) \geq\left|\exp \left(x y+|x|^{p-1}|y|^{q-1}\right)\right|
$$

and

$$
\begin{aligned}
& \frac{1}{p} \exp \left(|x|^{p}+|y|^{2}\right)+\frac{1}{q} \exp \left(|x|^{2}+|y|^{q}\right) \\
& \geq\left|\exp \left(x y+|x|^{\frac{2}{q}}|y|^{\frac{2}{p}}\right)\right|
\end{aligned}
$$

respectively, for any $x, y \in \mathbb{C}$ with $x, y \neq 0$.

(3) If we take the function $f(z)=\ln \left(\frac{1}{1-z}\right), z \in D(0,1)$, then from (2.15) and (2.16) we have

$$
\ln \left(1-|x|^{p}\right) \ln \left(1-|y|^{q}\right) \geq\left|\ln (1-x y) \ln \left(1-|x|^{p-1}|y|^{q-1}\right)\right|
$$

and

$$
\frac{1}{p} \ln \left(1-|x|^{p}\right) \ln \left(1-|y|^{2}\right)+\frac{1}{q} \ln \left(1-|x|^{2}\right) \ln \left(1-|y|^{q}\right) \geq\left|\ln (1-x y) \ln \left(1-|x|^{\frac{2}{q}}|y|^{\frac{2}{p}}\right)\right|,
$$


respectively, where $p>1, \frac{1}{p}+\frac{1}{q}=1$ and $x, y \neq 0$ with $x y,|x|^{2},|x|^{p},|x|^{\frac{1}{q}},|y|^{2},|y|^{q},|y|^{\frac{1}{p}} \in$ $D(0,1)$.

(4) If we consider the function $f(z)=\sin (z), z \in \mathbb{C}$, then we have $f_{A}(z)=\sinh (z), z \in \mathbb{C}$. Applying inequalities (2.15) and (2.16) to this function, we get

$$
\sinh \left(|x|^{p}\right) \sinh \left(|y|^{q}\right) \geq\left|\sin (x y) \sin \left(|x|^{p-1}|y|^{q-1}\right)\right|
$$

and

$$
\begin{aligned}
& \frac{1}{p} \sinh \left(|x|^{p}\right) \sinh \left(|y|^{2}\right)+\frac{1}{q} \sinh \left(|x|^{2}\right) \sinh \left(|y|^{q}\right) \\
& \quad \geq\left|\sin (x y) \sin \left(|x|^{\frac{2}{q}}|y|^{\frac{2}{p}}\right)\right|,
\end{aligned}
$$

respectively, where $p>1, \frac{1}{p}+\frac{1}{q}=1$ and $x, y \in \mathbb{C}$ with $x, y \neq 0$.

A similar result can be obtained for $\cosh (x)$ as well.

Theorem 3 Let $f(z)$ and $g(z)$ be as in Theorem 1 . Then one has the inequalities

$$
\begin{gathered}
\frac{1}{p^{2}} g_{A}\left(|x|^{2}\right) f_{A}\left(|y|^{q}\right)+\frac{1}{q} f_{A}\left(|x|^{p}\right) g_{A}\left(|y|^{2}\right) \\
\quad \geq\left|f\left(|x|^{p-1}|y|^{q-1}\right) g\left(|x|^{\frac{2}{p}}|y|^{\frac{2}{q}}\right)\right|
\end{gathered}
$$

and

$$
\frac{1}{p} g_{A}\left(|x|^{2}\right) f_{A}\left(|y|^{p}\right)+\frac{1}{q} f_{A}\left(|x|^{2}\right) g_{A}\left(|y|^{q}\right) \geq\left|f\left(|x|^{\frac{2}{q}} y\right) g\left(|x|^{\frac{2}{p}} y\right)\right| .
$$

Proof Follows from inequality (1.3) on choosing $x=\frac{|y|^{\frac{2}{q} k}}{|y|^{j}}, y=\frac{|x| \frac{2}{p} k}{|x|^{j}}$ and $x=|x|^{\frac{2}{q} j}|y|^{k}, y=$ $|x|^{\frac{2}{p} k}|y|^{j}$. That is, for any $i, j \in\{0,1,2, \ldots, n\}$, we have the following inequalities:

$$
\begin{aligned}
p|x|^{p j}|y|^{2 k}+q|x|^{2 k}|y|^{q j} & \geq p q|x|^{(p-1) j}|y|^{(q-1) j}|x|^{\frac{2}{p} k}|y|^{\frac{2}{q} k} \\
& =p q\left|\left(|x|^{(p-1)}|y|^{(q-1)}\right)^{j}\left(|x|^{\frac{2}{p}}|y|^{\frac{2}{q}}\right)^{k}\right|
\end{aligned}
$$

and

$$
\begin{aligned}
p|x|^{2 j}|y|^{q k}+q|x|^{2 k}|y|^{p j} & \geq p q|x|^{\frac{2}{q} j}|y|^{j}|x|^{\frac{2}{p} k}|y|^{k} \\
& =p q\left|\left(|x|^{\frac{2}{q}} y\right)^{j}\left(|x|^{\frac{2}{p}} y\right)^{k}\right|,
\end{aligned}
$$

respectively.

Repeating the same method as in Theorem 1 for (2.21) and (2.22), we deduce the desired inequalities, i.e., (2.19) and (2.20).

As a particular case of interest, we can state the following corollary.

Corollary 3 If $g(z)=f(z)$ in (2.19) and (2.20), then

$$
\frac{1}{p} f_{A}\left(|x|^{2}\right) f_{A}\left(|y|^{q}\right)+\frac{1}{q} f_{A}\left(|x|^{p}\right) f_{A}\left(|y|^{2}\right) \geq\left|f\left(|x|^{p-1}|y|^{q-1}\right) f\left(|x|^{\frac{2}{p}}|y|^{\frac{2}{q}}\right)\right|
$$


and

$$
f_{A}\left(|x|^{2}\right)\left[\frac{1}{p} f_{A}\left(|y|^{p}\right)+\frac{1}{q} f_{A}\left(|y|^{q}\right)\right] \geq\left|f\left(|x|^{\frac{2}{q}} y\right) f\left(|x|^{\frac{2}{p}} y\right)\right|,
$$

where $p>1, \frac{1}{p}+\frac{1}{q}=1$ and $x, y \neq 0$ with $|x|^{2},|x|^{p},|x|^{q},|y|^{2},|y|^{p},|y|^{q} \in D(0, R)$. In particular, if $y=x$ in (2.23) and (2.24), then we have

$$
f_{A}\left(|x|^{2}\right)\left[\frac{1}{p} f_{A}\left(|x|^{q}\right)+\frac{1}{q} f_{A}\left(|x|^{p}\right)\right] \geq\left|f\left(|x|^{2}\right) f\left(|x|^{p q-2}\right)\right|
$$

and

$$
f_{A}\left(|x|^{2}\right)\left[\frac{1}{p} f_{A}\left(|x|^{p}\right)+\frac{1}{q} f_{A}\left(|x|^{q}\right)\right] \geq\left|f\left(|x|^{\frac{2}{q}} x\right) f\left(|x|^{\frac{2}{p}} x\right)\right|
$$

for $x \neq 0,|x|^{2},|x|^{p},|x|^{q} \in D(0, R)$.

Inequalities (2.23) and (2.24) are also valuable sources of particular inequalities for complex numbers as will be outlined in the following.

(1) If we apply inequalities (2.23) and (2.24) to the function $f(z)=\frac{1}{1-z}, z \in D(0,1)$, then we get

$$
\begin{gathered}
\frac{1}{p\left(1-|x|^{2}\right)\left(1-|y|^{q}\right)}+\frac{1}{q\left(1-|x|^{p}\right)\left(1-|y|^{2}\right)} \\
\geq \frac{1}{\left|\left(1-|x|^{p-1}|y|^{q-1}\right)\left(1-|x|^{\frac{2}{p}}|y|^{\frac{2}{q}}\right)\right|}
\end{gathered}
$$

and

$$
\begin{gathered}
\frac{1}{1-|x|^{2}}\left(\frac{1}{p\left(1-|y|^{p}\right)}+\frac{1}{q\left(1-|y|^{q}\right)}\right) \\
\geq \frac{1}{\left|\left(1-|x|^{\frac{2}{q}} y\right)\left(1-|x|^{\frac{2}{p}} y\right)\right|},
\end{gathered}
$$

respectively, for any $x, y \in \mathbb{C}, x, y \neq 0$ with $|x|^{2},|y|^{2},|x|^{p},|y|^{q},|x|^{\frac{1}{p}},|x|^{\frac{1}{q}},|y|^{\frac{1}{p}},|y|^{\frac{1}{q}} \in D(0,1)$.

(2) If we apply inequalities (2.23) and (2.24) to the function $f(z)=\exp (z), z \in \mathbb{C}$, then we can state that

$$
\begin{gathered}
\frac{1}{p} \exp \left(|x|^{2}+|y|^{q}\right)+\frac{1}{q} \exp \left(|x|^{p}+|y|^{2}\right) \\
\geq\left|\exp \left(|x|^{p-1}|y|^{q-1}+|x|^{\frac{2}{p}}|y|^{\frac{2}{q}}\right)\right|
\end{gathered}
$$

and

$$
\begin{array}{r}
\exp \left(|x|^{2}\right)\left[\frac{1}{p} \exp \left(|y|^{p}\right)+\frac{1}{q} \exp \left(|y|^{q}\right)\right] \\
\geq\left|\exp \left[\left(|x|^{\frac{2}{q}}+|x|^{\frac{2}{p}}\right) y\right]\right|
\end{array}
$$

respectively, for $x, y \in \mathbb{C}, x, y \neq 0$. 
(3) If we apply the function $f(z)=\ln \left(\frac{1}{1-z}\right), z \in D(0,1)$, then from (2.23) and (2.24) we have

$$
\begin{aligned}
& \frac{1}{p} \ln \left(1-|x|^{2}\right) \ln \left(1-|y|^{q}\right)+\frac{1}{q} \ln \left(1-|x|^{p}\right) \ln \left(1-|y|^{2}\right) \\
& \geq\left|\ln \left(1-|x|^{p-1}|y|^{q-1}\right) \ln \left(1-|x|^{\frac{2}{p}}|y|^{\frac{2}{q}}\right)\right|
\end{aligned}
$$

and

$$
\begin{aligned}
& \ln \left(1-|x|^{2}\right)\left[\frac{1}{p} \ln \left(1-|y|^{p}\right)+\frac{1}{q} \ln \left(1-|y|^{q}\right)\right] \\
& \geq\left|\ln \left(1-|x|^{\frac{2}{q}} y\right) \ln \left(1-|x|^{\frac{2}{p}} y\right)\right|,
\end{aligned}
$$

respectively, for any $x, y \in \mathbb{C}, x, y \neq 0$ with $|x|^{2},|y|^{2},|x|^{p},|y|^{q},|x|^{\frac{1}{p}},|x|^{\frac{1}{q}},|y|^{\frac{1}{p}},|y|^{\frac{1}{q}} \in D(0,1)$.

(4) If we consider the function $f(z)=\sin (z), z \in \mathbb{C}$, then we have $f_{A}(z)=\sinh (z), z \in \mathbb{C}$. Applying inequalities (2.23) and (2.24) to this function, we get

$$
\begin{aligned}
& \frac{1}{p} \sinh \left(|x|^{2}\right) \sinh \left(|y|^{q}\right)+\frac{1}{q} \sinh \left(|x|^{p}\right) \sinh \left(|y|^{2}\right) \\
& \quad \geq\left|\sin \left(|x|^{p-1}|y|^{q-1}\right) \sin \left(|x|^{\frac{2}{p}}|y|^{\frac{2}{q}}\right)\right|
\end{aligned}
$$

and

$$
\sinh \left(|x|^{2}\right)\left[\frac{1}{p} \sinh \left(|y|^{p}\right)+\frac{1}{q} \sinh \left(|y|^{q}\right)\right] \geq\left|\sin \left(|x|^{\frac{2}{q}} y\right) \sin \left(|x|^{\frac{2}{p}} y\right)\right|,
$$

respectively, for any $x, y \in \mathbb{C}, x, y \neq 0$.

A similar result can be obtained for $\cosh (x)$ as well.

\section{Applications to special functions}

In this section, we give some inequalities for some special functions such as polylogarithm, hypergeometric, Bessel and modified Bessel functions for the first kind. Before that, we state here some basic concepts and definitions of those functions.

The polylogarithm $L i_{n}(z)$ is a function defined by the power series

$$
L i_{n}(z)=\sum_{k=1}^{\infty} \frac{z^{k}}{k^{n}}
$$

which converges absolutely for all complex values of the order $n$ and the argument $z$ where $|z|<1$. It is also known in the literature as Jonquiére's function. The special cases $z=-1,1$ reduce to $L i_{n}(1)=\zeta(n)$ and $L i_{n}(-1)=-\eta(n)$, where $\zeta$ and $\eta$ are the Riemann zeta function and Dirichlet eta function, respectively. When $n=1$, the first polylogarithm involves the ordinary logarithm, i.e., $L i_{1}(z)=-\ln (1-z)$, while the second

$$
L i_{2}=\sum_{k=1}^{\infty} \frac{z^{k}}{k^{2}}
$$

is called the dilogarithm or Spence's function. 
For other integer values of order $n$, the polylogarithm reduces to the ratio of a polynomial in $z$, for instance,

$$
\begin{aligned}
& L i_{0}(z)=\frac{z}{1-z}, \quad L i_{-1}(z)=\frac{z}{(1-z)^{2}}, \\
& L i_{-2}(z)=\frac{z(z+1)}{(1-z)^{3}}, \quad L i_{-3}(z)=\frac{z\left(1+4 z+z^{2}\right)}{(1-z)^{4}} .
\end{aligned}
$$

The hypergeometric function ${ }_{2} F_{1}(a, b ; c ; z)$ is defined for all $|z|<1$ by the series

$$
{ }_{2} F_{1}(a, b ; c ; z)=\sum_{n=0}^{\infty} \frac{(a)_{n}(b)_{n}}{(c)_{n}} \frac{z^{n}}{n !}
$$

where $a, b, c \in \mathbb{R}$ with $c \neq 0,-1,-2, \ldots$ and the $(t)_{n}, n \in\{0,1,2, \ldots\}$ is a Pochhammer symbol which is defined by

$$
(t)_{n}= \begin{cases}1 & \text { if } n=0 \\ t(t+1) \cdots(t+n-1) & \text { if } n>0\end{cases}
$$

Hypergeometric function (3.3) with particular arguments of $a, b$ and $c$ reduces to elementary functions. For instance,

$$
\begin{aligned}
& { }_{2} F_{1}(1,1 ; 1 ; z)={ }_{2} F_{1}(1,2 ; 2 ; z)=\frac{1}{1-z}, \\
& { }_{2} F_{1}(1,2 ; 1 ; z)=\frac{1}{(1-z)^{2}}, \\
& { }_{2} F_{1}(a, b ; b ; z)=\frac{1}{(1-z)^{a}}, \\
& { }_{2} F_{1}(1,1 ; 2 ; z)=\frac{1}{z} \ln \left(\frac{1}{1-z}\right), \\
& { }_{2} F_{1}(1,1 ; 2 ;-z)=\frac{1}{z} \ln (1+x) .
\end{aligned}
$$

Further, the Bessel functions of the first kind, denoted as $J_{\alpha}(z)$, are defined by the power series

$$
J_{\alpha}(z)=\sum_{k=0}^{\infty} \frac{(-1)^{k}}{k !(\alpha+k) !}\left(\frac{z}{2}\right)^{2 k+\alpha}
$$

for $\alpha, z \in \mathbb{C}$ with $|z|<1$. If $z$ is replaced by arguments $\pm i z$, then from (3.4) we have

$$
I_{\alpha}(z)=i^{-\alpha} J_{\alpha}(i z)=\sum_{k=0}^{\infty} \frac{1}{k !(\alpha+k) !}\left(\frac{z}{2}\right)^{2 k+\alpha}
$$

for $\alpha, z \in \mathbb{C}$ with $|z|<1$. These functions (3.5) are called the modified Bessel functions of the first kind.

It is clearly seen that from (3.1), (3.3), (3.4) and (3.5), that is, $L i_{n}(z),{ }_{2} F_{1}(a, b ; c ; z), J_{\alpha}(z)$ and $I_{\alpha}(z)$ are power series with real coefficients and convergent on the open disk $D(0,1)$. 
Therefore, all the results in the above section hold true. For instance, from (2.15) we have the following corollaries.

Corollary 4 If $L i_{n}(z)$ is the polylogarithm function, then we have

$$
\operatorname{Li}_{n}\left(|x|^{p}\right) L i_{n}\left(|y|^{q}\right) \geq\left|L i_{n}(x y) L i_{n}\left(|x|^{p-1}|y|^{q-1}\right)\right|
$$

for any $x, y \in \mathbb{C}, x, y \neq 0$ with $x y,|x|^{p},|y|^{q} \in D(0,1)$ and $p, q>1, \frac{1}{p}+\frac{1}{q}=1$.

In particular, if $n=0$ in (3.6), then we have the following inequality:

$$
\left.|1-x y||1-| x\right|^{p-1}|y|^{q-1} \mid \geq\left(1-|x|^{p}\right)\left(1-|y|^{q}\right)
$$

for all $x, y \neq 0, x y,|x|^{p},|y|^{q} \in D(0,1)$ and $p, q>1$ with $\frac{1}{p}+\frac{1}{q}=1$.

If we take $n=1$ in (3.6), then we get inequality (2.18) for all $x, y \neq 0$ with $x y,|x|^{p},|y|^{q} \in$ $D(0,1)$ and $p, q>1, \frac{1}{p}+\frac{1}{q}=1$.

Also, if we choose in (3.6) $n=2$, then we obtain

$$
L i_{2}\left(|x|^{p}\right) L i_{2}\left(|y|^{q}\right) \geq\left|L i_{2}(x y) L i_{2}\left(|x|^{p-1}|y|^{q-1}\right)\right|
$$

for any $x, y \neq 0, x y,|x|^{p},|y|^{q} \in D(0,1)$ and $p>1$ with $\frac{1}{p}+\frac{1}{q}=1$. Li $2(z)$ is the dilogarithm function which is defined in (3.2).

Corollary 5 If ${ }_{2} F_{1}(a, b ; c ; z)$ is a hypergeometric function, then for any $a, b, c \in \mathbb{R}$, we have

$$
\begin{aligned}
& { }_{2} F_{1}\left(a, b ; c ;|x|^{p}\right){ }_{2} F_{1}\left(a, b ; c ;|y|^{q}\right) \\
& \quad \geq\left|{ }_{2} F_{1}(a, b ; c ; x y){ }_{2} F_{1}\left(a, b ; c ;|x|^{p-1}|y|^{q-1}\right)\right|,
\end{aligned}
$$

where $x, y \neq 0$ with $x y,|x|^{p},|y|^{q} \in D(0,1)$ and $p>1, \frac{1}{p}+\frac{1}{q}=1$.

In particular, if we choose $a=1, c=b$ in (3.7), then we get inequality (2.17). Also, if we choose $a=b=1, c=2$, then inequality (3.7) reduces to (2.18).

Corollary 6 If $J_{\alpha}(z)$ and $I_{\alpha}(z)$ are the Bessel and modified Bessel functions of the first kind, respectively, then for any $\alpha, x, y \in \mathbb{C}$, we have

$$
I_{\alpha}\left(|x|^{p}\right) I_{\alpha}\left(|y|^{q}\right) \geq\left|J_{\alpha}(x y) J_{\alpha}\left(|x|^{p-1}|y|^{q-1}\right)\right|,
$$

where $x, y \neq 0, x y,|x|^{p},|y|^{q} \in D(0,1)$ and $p>1$ with $\frac{1}{p}+\frac{1}{q}=1$.

In particular, if $\alpha=0$ in (3.8), then for $p, q>1$ with $\frac{1}{p}+\frac{1}{q}=1$, we get

$$
J_{0}\left(i|x|^{p}\right) J_{0}\left(i|y|^{q}\right) \geq\left|J_{0}(x y) J_{0}\left(|x|^{p-1}|y|^{q-1}\right)\right|,
$$

where $J_{0}(z)=\sum_{k=0}^{\infty} \frac{(-1)^{k}}{(k !)^{2}}\left(\frac{z}{2}\right)^{2 k}$.

Other inequalities involving the polylogarithm, hypergeometric, Bessel and modified Bessel functions can be found in the literature (see [23-28] and references therein). 


\section{Competing interests}

The authors declare that they have no competing interests.

\section{Authors' contributions}

The first author Al is currently a PhD student under supervision of the second author SSD and the third author MD is the co-supervisor. They jointly worked on deriving the results. All authors read and approved the final manuscript.

\section{Author details}

${ }^{1}$ School of Engineering and Science, Victoria University, P.O. Box 14428, Melbourne, 8001, Australia. ${ }^{2}$ School of Computational and Applied Mathematics, University of the Witwatersrand, Private Bag 3, Johannesburg, 2050, South Africa. ${ }^{3}$ School of Mathematical Sciences, Faculty of Science and Technology, Universiti Kebangsaan Malaysia, Bangi, Selangor 43600, Malaysia.

Received: 6 August 2012 Accepted: 13 June 2013 Published: 3 July 2013

\section{References}

1. Beckenbach, EF, Bellman, R: Inequalities. Springer, Berlin (1961)

2. Tolsted, E: An elementary derivation of the Cauchy, Hö Ider and Minkowski inequalities from Young's inequality. Math. Mag. 37, 2-12 (1964)

3. Marshall, AW, Olkin, I: Inequalities: Theory of Majorization and Its Applications. Academic Press, New York/London (1979)

4. Rudin, W: Real and Complex Analysis, 3rd edn. McGraw-Hill, New York (1987)

5. Young, WH: On classes of summable functions and their Fourier series. Proc. R. Soc. Lond. A 87(594), 225-229 (1912)

6. Cerone, P: On Young's inequality and its reverse for bounding the Lorenz curve and Gini mean. J. Math. Inequal. 3(3), 369-381 (2009)

7. Hong, FH, Yeh, CC, Yu, SL, Hong, CH: Young's inequality and related results on time scales. Appl. Math. Lett. 18, 983-988 (2005)

8. Mitrinovic, DS, Pečaric, JE, Fink, AM: Classical and New Inequalities in Analysis. Kluwer Academic, Dordrecht (1993)

9. Hardy, G, Littlewood, JE, Pólya, G: Inequalities. Cambridge University Press, Cambridge (1952)

10. Abramovich, S, Mond, B, Pecaric, JE: Sharpening Hölder's inequality. J. Math. Anal. Appl. 196, 1131-1134 (1995)

11. Carroll, JA, Cordner, R, Evelyn, CJA: A new extension of Hölder's inequality. Enseign. Math. 16, 69-71 (1970)

12. Daykin, DE, Eliezer, CJ: Generalization of Hölder's and Minkowski's inequalities. Proc. Camb. Philos. Soc. 64, 1023-1027 (1968)

13. He, WS: Generalization of a sharp Hölder's inequality and its application. J. Math. Anal. Appl. 332, 741-750 (2007)

14. Kim, YI, Yang, X: Generalizations and refinements of Hölder's inequality. Appl. Math. Lett. 25, 1094-1097 (2012)

15. Mitrinovic, DS, Pečaric, JE: On an extension of Hölder's inequality. Boll. Un. Mat. Ital. A (7) 4, 405-408 (1990)

16. Kwon, EG: Extension of Hölder's inequality (1). Bull. Aust. Math. Soc. 51, 369-375 (1995)

17. Qiang, H, Hu, Z: Generalizations of Hölder's and some related inequalities. Comput. Math. Appl. 61, $392-396$ (2011)

18. Yang, X: Hölder's inequality. Appl. Math. Lett. 16, 897-903 (2003)

19. Yang, X: A generalization of Hölder's inequality. J. Math. Anal. Appl. 247, 328-330 (2000)

20. Yang, X: Refinement of Hölder's inequality and application to Ostrowski inequality. Appl. Math. Comput. 138, 455-461 (2003)

21. Dragomir, SS, Sándor, J: Some generalizations of Cauchy-Bunyakovsky-Schwarz's inequality. Gaz. Mat. Metod. (Bucharest) 11, 104-109 (1990) (in Romanian)

22. Dragomir, SS: Discrete Inequalities of the Cauchy-Bunyakovsky-Schwarz Type. Nova Publ., New York (2004)

23. Baricz, A: Functional inequalities involving Bessel and modified Bessel functions of the first kind. Expo. Math. 26 279-293 (2008)

24. Barnard, RW, Kendall, KC: On inequalities for hypergeometric analogues of the arithmetic-geometric mean. J. Inequal. Pure Appl. Math. 8(3), 1-12 (2007)

25. He, B, Yang, B: On a Hilbert-type inequality with a hypergeometric function. Commun. Math. Anal. 9(1), 84-92 (2010)

26. Jemai, MM: A main inequality for several special functions. Comput. Math. Appl. 60, 1280-1289 (2010)

27. Yadava, SR, Singh, B: Certain inequalities involving special functions. Proc. Natl. Acad. Sci. India, Sect. a Phys. Sci. 57(3), 324-328 (1987)

28. Zhu, L: Jordan type inequalities involving the Bessel and modified Bessel functions. Comput. Math. Appl. 59, 724-736 (2010)

doi:10.1186/1029-242X-2013-314

Cite this article as: Ibrahim et al.: Power series inequalities via Young's inequality with applications. Journal of Inequalities and Applications 2013 2013:314. 